\title{
Characterization of near-room-temperature superconductivity in yttrium
}

\section{superhydrides}

\author{
E. F. Talantsev ${ }^{1,2^{*}}$ \\ ${ }^{1}$ M.N. Mikheev Institute of Metal Physics, Ural Branch, Russian Academy of Sciences, \\ 18, S. Kovalevskoy St., Ekaterinburg, 620108, Russia \\ ${ }^{2}$ NANOTECH Centre, Ural Federal University, 19 Mira St., Ekaterinburg, 620002, \\ Russia
}

*E-mail: evgeny.talantsev@imp.uran.ru

\begin{abstract}
Recently, Troyan et al (2019 arXiv:1908.01534) and Kong et al (2019 arXiv:1909.10482) extended near-room-temperature superconductors family by new yttrium superhydride polymorphs, $\mathrm{YH}_{\mathrm{n}}(\mathrm{n}=4,6,7,9)$, which exhibit superconducting transition temperatures in the range of $T_{\mathrm{c}}=210-243 \mathrm{~K}$ at pressure of $P=160-255 \mathrm{GPa}$. In this paper, temperature dependent upper critical field data, $B_{\mathrm{c} 2}(T)$, for highly-compressed mixture of $\mathrm{YH}_{4}+\mathrm{YH}_{6}$ phases (reported by Kong et al 2019 arXiv:1909.10482) is analysed to deduce the ratio of $T_{\mathrm{c}}$ to the Fermi temperature, $T_{\mathrm{F}}$. Our analysis shows that in all considered scenarios the $\mathrm{YH}_{4}+\mathrm{YH}_{6}$ mixture has the ratio $0.01 \leq T_{\mathrm{c}} / T_{\mathrm{F}} \leq 0.04$. As the result, $\mathrm{YH}_{4}+\mathrm{YH}_{6}$ falls in the unconventional superconductors band in the Uemura plot. It is also found that the characteristic temperature of the order parameter amplitude fluctuations, $T_{\text {fluc }}$, in the $\mathrm{YH}_{4}+\mathrm{YH}_{6}$ mixture is only several percent above observed $T_{\mathrm{c}}$, and thus the superconducting transition in yttrium superhydride polymorphs is fundamentally limited by thermodynamics fluctuations.
\end{abstract}




\section{Introduction}

Recently, Troyan et al [1] and Kong et al [2] reported on the discovery of new superhydride polymorphs of yttrium, $\mathrm{YH}_{\mathrm{n}}(\mathrm{n}=4,6,7,9)$, which exhibit superconducting transition at $T_{\mathrm{c}}=210-243 \mathrm{~K}$ to be subjected to pressure of $P=160-255 \mathrm{GPa}$. As far as pure hydrogen, pressurized to $P=440 \mathrm{GPa}$, does not show any evidences for superconducting transition down to $T=4.2 \mathrm{~K}$ [3], the near-room-temperature (NRT) hydrogen-rich superconductors family [4],[5],[6],[7] has been extended by new $\mathrm{YH}_{\mathrm{n}}$ polymorphs [1],[2]. Kong et al [2] attribute the highest observed $T_{\mathrm{c}}=237-243 \mathrm{~K}$ for $\mathrm{P} 6_{3} / m m c-\mathrm{YH}_{9}$ phase, and this experimental findings are well supported by the first principles calculation studies [8],[9],[10],[11].

In this paper we analyse temperature dependent upper critical field data, $B_{\mathrm{c} 2}(T)$ (reported by Kong et al [2] for the mixture of $\mathrm{YH}_{4}+\mathrm{YH}_{6}$ phases) with the purpose to classify the superconductivity in this NRT superconductor and to deduce the characteristic temperature of thermodynamic fluctuations, $T_{\text {fluc }}$, in this highly-compressed superhydride of yttrium.

\section{Ground state coherence length}

Kong et al [2] in their Figure 1(d) reported the temperature dependent magnetoresistance, $R(T, B)$, for Sample 5 pressurised at $P=185 \mathrm{GPa}$, which contents the mixture of $\mathrm{YH}_{4}+\mathrm{YH}_{6}$ phases $\left(T_{\mathrm{c}} \sim 214 \mathrm{~K}\right)$. By utilising the criterion of $75 \%$ of normal state resistance, $R(T) / R_{\text {norm }}=$ 0.75 , we deduce the upper critical field, $B_{\mathrm{c} 2}(T)$, dataset and fit it to four models:

1. Gorter-Casimir model (GC model, Fig. 1(a)) [12]:

$$
B_{c 2}(T)=\frac{\phi_{0}}{2 \cdot \pi \cdot \xi^{2}(0)} \cdot\left(1-\left(\frac{T}{T_{c}}\right)^{2}\right)
$$

where $\phi_{0}=2.068 \cdot 10^{-15} \mathrm{~Wb}$ is magnetic flux quantum.

2. Gorkov model (Fig. 1(b)) [13]: 


$$
B_{c 2}(T)=\frac{\phi_{0}}{2 \cdot \pi \cdot \xi^{2}(0)} \cdot\left(\frac{1.77-0.43 \cdot\left(\frac{T}{T_{C}}\right)^{2}+0.07 \cdot\left(\frac{T}{T_{C}}\right)^{4}}{1.77}\right) \cdot\left[1-\left(\frac{T}{T_{C}}\right)^{2}\right]
$$

3. Werthamer-Helfand-Hohenberg model (WHH model, (Fig. 1(c)) [14]:

$$
B_{c 2}(0)=\frac{\phi_{0}}{2 \cdot \pi \cdot \xi^{2}(0)}=-0.693 \cdot T_{c} \cdot\left(\frac{d B_{c 2}(T)}{d T}\right)_{T \sim T_{C}},
$$

4. Baumgartner et al model (B-WHH model, Fig. 1(c)) [15]:

$$
B_{c 2}(T)=\frac{\phi_{0}}{2 \cdot \pi \cdot \xi^{2}(0)} \cdot\left(\frac{\left(1-\frac{T}{T_{C}}\right)-0.153 \cdot\left(1-\frac{T}{T_{C}}\right)^{2}-0.152 \cdot\left(1-\frac{T}{T_{C}}\right)^{4}}{0.693}\right)
$$

Result of fits to these four models are shown in Fig. 1 and Table 1.

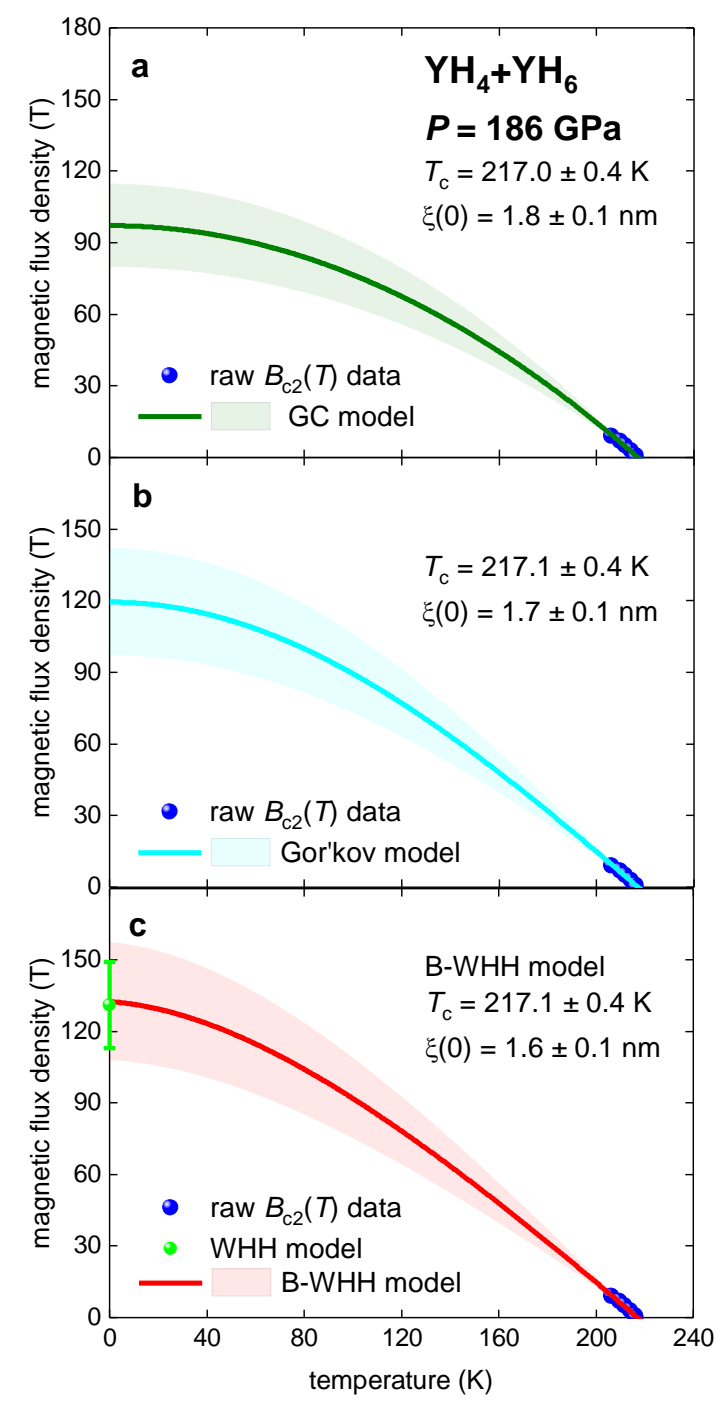

Figure 1. Superconducting upper critical field, $B_{\mathrm{c} 2}(T)$, data and fits to four models (Eqs. 1-4) for $\mathrm{YH}_{4}+\mathrm{YH}_{6}$ mixture (Sample 5 [2]) compressed at pressure of $P=186 \mathrm{GPa}$. (a) fit to GC model, the fit quality is $R=0.982$. (b) fit to Gor'kov model, $R=0.981$. (c) fit to WHH and BWHH models, for latter $R=0.981 .95 \%$ confidence bars are shown. 
Table I. Deduced and calculated parameters for the mixture of $\mathrm{YH}_{4}+\mathrm{YH}_{6}$ compressed at $P=$ $186 \mathrm{GPa}$ (Sample 5 [2]). Deduced critical temperature for all models (Eqs. 1-4) is $T_{\mathrm{c}}=217$ $\mathrm{K}$. Assumed charge effective mass is $m_{e f f}^{*}=2.76 \cdot m_{e}$ [11]. Smallest and largest values for $\frac{T_{C}}{T_{F}}, \frac{T_{C}}{T_{\text {fluc,phase }}}$ and $\frac{T_{C}}{T_{\text {fluc,amp }}}$ are marked in bold.

\begin{tabular}{|c|c|c|c|c|c|c|c|c|c|}
\hline Model & $\begin{array}{l}\text { Deduced } \\
\xi(0)(\mathrm{nm})\end{array}$ & $\begin{array}{l}\text { Assumed } \\
\frac{2 \cdot \Delta(0)}{k_{B} \cdot T_{C}}\end{array}$ & $\begin{array}{c}T_{\mathrm{F}}\left(10^{3}\right. \\
\mathrm{K})\end{array}$ & $T_{\mathrm{d}} / T_{\mathrm{F}}$ & $\begin{array}{c}\text { Assumed } \\
\kappa\end{array}$ & $\begin{array}{l}T_{\text {fluc,phase }} \\
(\mathrm{K})\end{array}$ & $\begin{array}{l}T_{\text {fluc,amp }} \\
(\mathrm{K})\end{array}$ & $T_{\mathrm{c}} / T_{\text {fluc,phase }}$ & $T_{\mathrm{c}} / T_{\text {fluc,amp }}$ \\
\hline \multirow{2}{*}{ GC } & \multirow[t]{2}{*}{$1.8 \pm 0.1$} & 3.53 & $\begin{array}{l}7.2 \pm \\
0.9\end{array}$ & $\begin{array}{c}0.030 \pm \\
0.003\end{array}$ & 60 & $3750 \pm 190$ & $\begin{array}{c}1010 \pm \\
55\end{array}$ & $0.058 \pm 0.003$ & $0.22 \pm 0.01$ \\
\hline & & 5.47 & $\begin{array}{c}17.4 \pm \\
2.0\end{array}$ & $\begin{array}{c}0.013 \pm \\
0.02\end{array}$ & 120 & $940 \pm 50$ & $252 \pm 13$ & $0.23 \pm 0.01$ & $0.86 \pm 0.05$ \\
\hline \multirow{2}{*}{ Gor'kov } & \multirow[t]{2}{*}{$1.7 \pm 0.1$} & 3.53 & $\begin{array}{c}6.5 \pm \\
0.7 \\
\end{array}$ & $\begin{array}{c}0.034 \pm \\
0.004 \\
\end{array}$ & 60 & $3970 \pm 220$ & $\begin{array}{c}1070 \pm \\
60 \\
\end{array}$ & $0.055 \pm 0.003$ & $0.20 \pm 0.02$ \\
\hline & & 5.47 & $\begin{array}{c}15.5 \pm \\
1.9\end{array}$ & $\begin{array}{c}0.014 \pm \\
0.001\end{array}$ & 120 & $994 \pm 45$ & $267 \pm 15$ & $0.22 \pm 0.01$ & $0.81 \pm 0.05$ \\
\hline \multirow{2}{*}{ B-WHH } & \multirow[t]{2}{*}{$1.6 \pm 0.1$} & 3.53 & $\begin{array}{l}5.7 \pm \\
0.9\end{array}$ & $\begin{array}{c}0.038 \pm \\
0.003\end{array}$ & 60 & $4220 \pm 250$ & $\begin{array}{c}1130 \pm \\
60\end{array}$ & $0.051 \pm 0.005$ & $0.19 \pm 0.01$ \\
\hline & & 5.47 & $\begin{array}{c}13.7 \pm \\
1.8\end{array}$ & $\begin{array}{c}0.016 \pm \\
0.002\end{array}$ & 120 & $1060 \pm 65$ & $284 \pm 17$ & $0.21 \pm 0.01$ & $0.77 \pm 0.04$ \\
\hline
\end{tabular}

\section{III. $\mathrm{YH}_{4}+\mathrm{YH}_{6}$ mixture in Uemura plot}

From deduced $\xi(0)$ values (Fig. 1 and Table 1), we calculated the Fermi temperature, $T_{\mathrm{F}}$, for $\mathrm{YH}_{4}+\mathrm{YH}_{6}$ mixture by utilising standard approach of Bardeen-Cooper-Schrieffer theory [16] (details can be find elsewhere [17]):

$$
T_{F}=\frac{\varepsilon_{F}}{k_{B}}=\frac{\pi^{2}}{8} \cdot m_{e f f}^{*} \cdot \xi^{2}(0) \cdot\left(\frac{\alpha \cdot k_{B} \cdot T_{C}}{\hbar}\right)^{2},
$$

where $\alpha=\frac{2 \cdot \Delta(0)}{k_{B} \cdot T_{C}}, \Delta(0)$ is the amplitude of the ground state energy gap, $\varepsilon_{\mathrm{F}}$ is the Fermi energy, $\hbar=h / 2 \pi$ is reduced Planck constant, $k_{\mathrm{B}}$ is the Boltzmann constant, $m_{e f f}^{*}$ is the charge carrier effective mass $\left(m_{\text {eff }}^{*}=2.73 \cdot m_{e}\right.$ for $\mathrm{YH}_{6}$ at $\left.P=200-350 \mathrm{GPa}[11]\right)$.

For NRT superconductors $\alpha=3.53-5.47$, where the lower bound is reported for $\mathrm{H}_{3} \mathrm{~S}$ $[17,18]$ and the upper bond reported for $\mathrm{YH}_{\mathrm{x}}[1]$. Based on the fact that critical temperatures for $\mathrm{YH}_{4}+\mathrm{YH}_{6}$ mixture deduced by all four models are very close to each other, $T_{\mathrm{c}}=217.1 \pm$ $0.4 \mathrm{~K}$, in Table I we show only the $T_{\mathrm{c}} / T_{\mathrm{F}}$ ratios. 
As the result, the mixture of $\mathrm{YH}_{4}+\mathrm{YH}_{6}(P=185 \mathrm{GPa})$ in all considered scenarios (Table 1) has $0.01 \leq T_{\mathrm{c}} / T_{\mathrm{F}} \leq 0.04$ and falls in unconventional superconductors band of the Uemura plot $[19,20]$ in close proximity to other NRT counterparts [17],[21],[22] (Fig. 2).

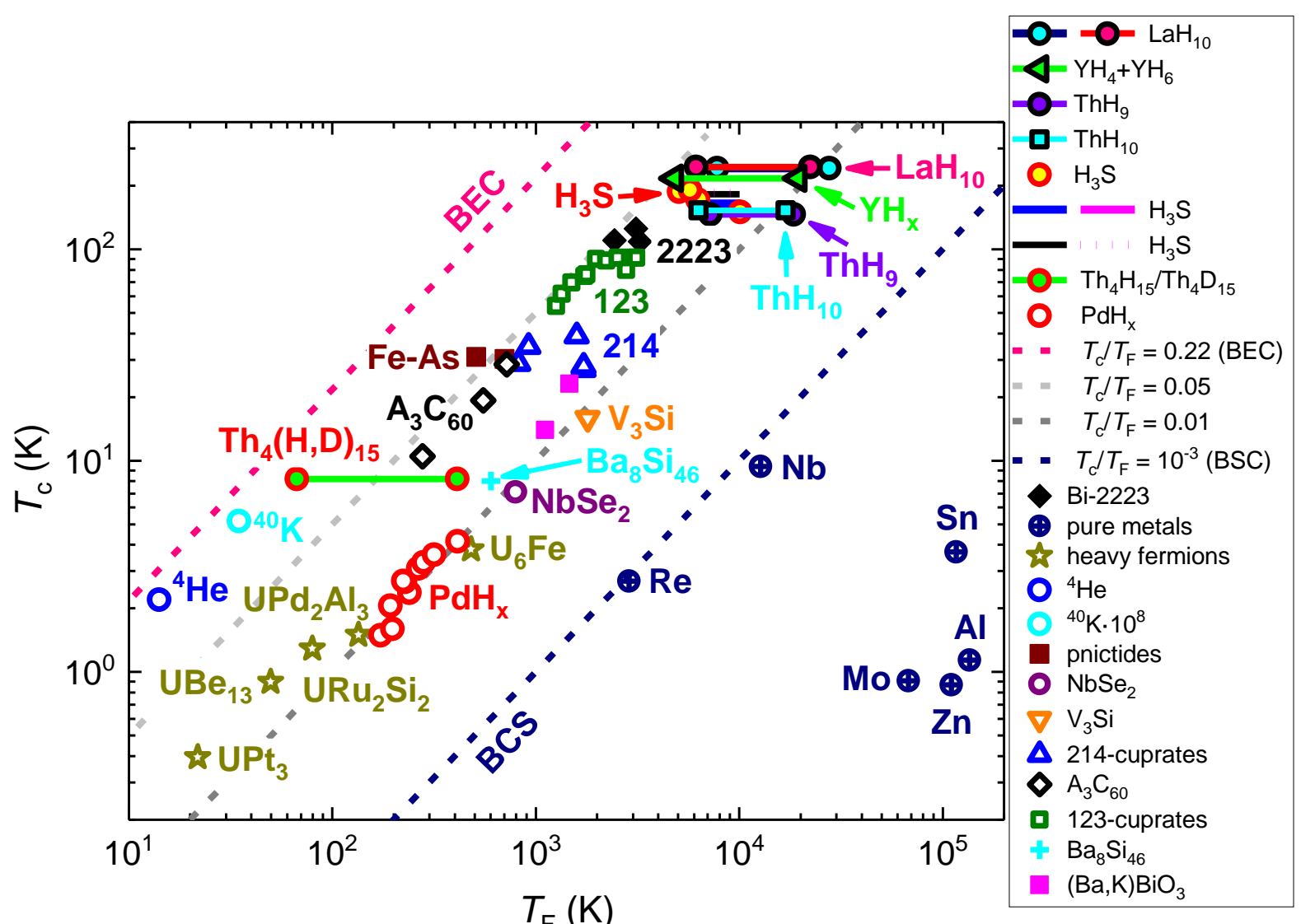

Figure 2. A plot of $T_{\mathrm{c}}$ versus $T_{\mathrm{F}}$ obtained for most representative superconducting families including $\mathrm{PdH}_{\mathrm{x}}, \mathrm{Th}_{4} \mathrm{H}_{15} / \mathrm{Th}_{4} \mathrm{D}_{15}, \mathrm{ThH}_{9}, \mathrm{ThH}_{10}, \mathrm{H}_{3} \mathrm{~S}, \mathrm{LaH}_{10}$, and the $\mathrm{YH}_{4}+\mathrm{YH}_{6}$ mixture (Sample 5 [2]). Other data was taken from [17],[19-22]. BEC and BCS lines are shown for clarity.

\section{Thermodynamic fluctuations of order parameters of $\mathrm{YH}_{4}+\mathrm{YH}_{6}$ mixture}

Both research groups [1,2] pointed out that experimental $T_{\mathrm{c}}$ values for $\mathrm{YH}_{\mathrm{x}}$ superhydrides are about $40-50 \mathrm{~K}$ lower than values predicted by the first principles calculations. One possible reason for observed $T_{\mathrm{c}}$ suppression can be originated by thermodynamic fluctuations of the order parameter $[23,24]$. There are two characteristic temperatures for thermodynamic fluctuations in superconductors, one is the phase order fluctuations temperature [23]: 


$$
T_{\text {fluc,phase }}=\frac{0.55 \cdot \phi_{0}^{2}}{\pi^{3 / 2} \cdot \mu_{0} \cdot k_{B}} \cdot \frac{1}{\kappa^{2} \cdot \xi(0)}
$$

where $\kappa=\lambda(0) / \xi(0)$ is Ginzburg-Landau parameter, and $\lambda(0)$ is the ground state London penetration depth.

The second is the characteristic temperature for the amplitude order parameter fluctuations [24]:

$$
T_{f l u c, a m p}=\frac{\phi_{0}^{2}}{12 \cdot \pi \cdot \mu_{0} \cdot k_{B}} \cdot \frac{1}{\kappa^{2} \cdot \xi(0)}
$$

Calculated values for $T_{\text {fluc,phase }}$ and $T_{\text {fluc,amp }}$ are shown in Table I, where the value of $\kappa=$ 60-120 covers expected range for majority of high-temperature superconductors [17],[21],[25,26]. It can be seen, that in some scenarios $T_{\mathrm{c}} / T_{\text {fluc,amp }} \sim 0.9$, which means that observed in experiment the suppression in $T_{\mathrm{c}}$ for the $\mathrm{YH}_{4}+\mathrm{YH}_{6}$ mixture of superhydrides can be explained by fundamental thermodynamic fluctuations.

\section{Results}

In result, in this paper the mixture of NRT superconductors $\mathrm{YH}_{4}+\mathrm{YH}_{6}$ has been classified as unconventional superconductor which is nicely matched the location of other NRT superhydrides in the Uemura plot. It is also shown the thermodynamic fluctuations of the order parameter amplitude is dominating factor which limits superconducting transition temperature in superhydrides of yttrium.

\section{Acknowledgement}

The author thanks financial support provided by the state assignment of Minobrnauki of Russia (theme "Pressure" No. AAAA-A18-118020190104-3) and by Act 211 Government of the Russian Federation, contract No. 02.A03.21.0006. 


\section{References}

[1] Troyan I A, et al. 2019 Synthesis and superconductivity of yttrium hexahydride $\operatorname{Im} \overline{3 m}$ $\mathrm{YH}_{6}$ arXiv:1908.01534

[2] Kong P P, et al. 2019 Superconductivity up to $243 \mathrm{~K}$ in yttrium hydrides under high pressure arXiv:1909.10482

[3] Eremets M I, Drozdov A P, Kong P P and Wang H 2019 Semimetallic molecular hydrogen at pressure above 350 GPa Nature Physics 15 1246-1249

[4] Drozdov A P, Eremets M I, Troyan I A, Ksenofontov V, Shylin S I 2015 Conventional superconductivity at 203 kelvin at high pressures in the sulfur hydride system Nature $\mathbf{5 2 5} 73$ 76

[5] Somayazulu M, Ahart M, Mishra A K, Geballe Z M, Baldini M, Meng Y, Struzhkin V V and R. J. Hemley R J 2019 Evidence for superconductivity above $260 \mathrm{~K}$ in lanthanum superhydride at megabar pressures Phys. Rev. Lett. 122027001

[6] Drozdov A P, et al. Superconductivity at $250 \mathrm{~K}$ in lanthanum hydride under high pressures Nature 569 528-531 (2019)

[7] Semenok D V, Kvashnin A G, Ivanova A G, Svitlyk V, Fominski V Yu, Sadakov A V, Sobolevskiy O A, Pudalov V M, Troyan I A and Oganov A R 2019 Materials Today, in press DOI: $10.1016 /$ j.mattod.2019.10.005

[8] Li Y, Hao J, Liu H, Tse J S, Wang Y and Ma Y 2015 Pressure-stabilized superconductive yttrium hydrides Scientific Reports 509948

[9] Peng F, et al. 2017 Hydrogen clathrate structures in rare earth hydrides at high pressures: Possible route to room-temperature superconductivity Phys. Rev. Lett. 119107001

[10] Liu H, Naumov I I, Hoffmann R, Ashcroft N W and Hemley R J 2017 Potential high- $T_{\mathrm{c}}$ superconducting lanthanum and yttrium hydrides at high pressure PNAS 114 6990-6995

[11] Heil C, di Cataldo S, Bachelet G B and Boeri L 2019 Superconductivity in sodalite-like yttrium hydride clathrates Physical Review B 99 220502(R)

[12] Gorter C J and Casimir H 1934 On supraconductivity I Physica 1 306-320

[13] Gor'kov L P 1960 The critical supercooling field in superconductivity theory Soviet Physics JETP 10 593-599

[14] Werthamer N R, Helfand E and Hohenberg P C 1966 Temperature and purity dependence of the superconducting critical field, $H_{\mathrm{c} 2}$. III. Electron spin and spin-orbit effects Phys. Rev. 147 295-302

[15] Baumgartner T, Eisterer M, Weber H W, Fluekiger R, Scheuerlein C, Bottura L 2014

Effects of neutron irradiation on pinning force scaling in state-of-the-art $\mathrm{Nb}_{3} \mathrm{Sn}$ wires

Supercond. Sci. Technol. 27015005

[16] Bardeen J, Cooper L N, Schrieffer J R 1957 Theory of superconductivity Phys. Rev.

108, $1175-1204$

[17] Talantsev E F 2019 Classifying superconductivity in compressed $\mathrm{H}_{3} \mathrm{~S}$ Modern Physics Letters B 331950195

[18] Kaplan D and Imry Y 2018 High-temperature superconductivity using a model of hydrogen bonds Proc. Nat. Acad. Sci. 115 5709-5713

[19] Uemura Y J 2004 Condensation, excitation, pairing, and superfluid density in high- $T_{\mathrm{c}}$ superconductors: the magnetic resonance mode as a roton analogue and a possible spinmediated pairing J. Phys.: Condens. Matter 16 S4515-S4540

[20] Uemura Y J 2019 Dynamic superconductivity responses in photoexcited optical conductivity and Nernst effect Phys. Rev. Materials 3104801

[21] Talantsev E F 2019 Classifying hydrogen-rich superconductors Materials Research Express 6106002 
[22] Talantsev E F and Mataira R C 2019 Classifying superconductivity in ThH-ThD superhydrides/superdeuterides arXiv:1910.00922v3

[23] Emery V J and Kivelson S A 1995 Importance of phase fluctuations in superconductors with small superfluid density Nature 374 434-437

[24] Bulaevskii L N, Ginzburg V L, Sobyanin A A 1988 Macroscopic theory of superconductors with small coherence length Physica C 152 378-388

[25] Hosono H et. al. 2015 Exploration of new superconductors and functional materials, and fabrication of superconducting tapes and wires of iron pnictides Sci. Technol. Adv.

Mater. 16033503

[26] Hänisch J et al. 2019 Fe-based superconducting thin films - Preparation and tuning of superconducting properties Supercond. Sci. Technol. 32093001 